\title{
Local Frequency Restoration for Droop-Controlled Parallel Inverters in Islanded Microgrids
}

\author{
Juan M. Rey, Carlos Xavier Rosero, Manel Velasco, Pau Martí, Jaume Miret, Member, IEEE, and Miguel Castilla
}

\begin{abstract}
In islanded microgrids, voltage source inverters working in parallel are expected to provide regulation of the local frequency while granting active power sharing. This paper presents a local control approach at each inverter based on an event-driven operation of a parameter-varying filter. It ensures perfect active power sharing and controllable accuracy for frequency restoration without requiring the exchange of control data between inverters over the communication network. The paper includes stability analysis and design guidelines for the control parameters using a modeling approach that considers the interaction between inverters. Selected experimental results on a three-inverter laboratory microgrid corroborate the effectiveness of the proposed control scheme, and outlines its advantages with respect to previous similar schemes and the performance cost that implies not using communications.
\end{abstract}

Index Terms-Microgrids, islanded mode, power sharing, droop control, frequency restoration, parameter-varying filter, local operation, event-driven operation

\section{INTRODUCTION}

The parallel operation of voltage source inverters (VSI) in an islanded microgrid (MG) has as a primary goal an even distribution of system load between them, which can be achieved by the droop control method [1]. The frequency deviation inherently induced by the droop method can be solved by diverse restoration strategies. Many solutions (see [2][15] to name a few) require exchanging control data between VSIs over a communication network to achieve frequency restoration. The traditional approach to restore the frequency is to apply a centralized structure based on communications where a central unit collects information of all the droopcontrolled units, executes a standard PI (proportional-integral) control, and sends back the computed control actions. Even knowing that accurate performance is easily achieved, this approach is sensitive to failures, leading to a single point of failure. To overcome this limitation, recent approaches are inspired in the decentralized control concept, making a

J.M. Rey is with Escuela de Ingenierías Eléctrica, Electrónica y de Telecomunicaciones (E3T), Universidad Industrial de Santander (UIS), 680002 Bucaramanga, Colombia, and with the Electronic Engineering Department, Technical University of Catalonia, Av. Victor Balaguer s/n, 08800 Vilanova i la Geltrú, Spain, email:juanmrey@uis.edu.co

C.X. Rosero, M. Velasco, and P. Martí are with the Automatic Control Department, Technical University of Catalonia, Pau Gargallo 5, 08028 Barcelona, Spain, email: carlos.xavier.rosero@gmail.com, \{manel.velasco,pau.marti\}@upc.edu

J. Miret and M. Castilla are with the Electronic Engineering Department, Technical University of Catalonia, Av. Victor Balaguer s/n, 08800 Vilanova i la Geltrú, Spain, email:\{jaume.miret,miquel.castilla\}@upc.edu

This work has been supported by the Ministry of Economy and Competitiveness of Spain and the European Regional Development Fund (FEDER) under the project ENE2015-64087-C2-1-R. different use of the communication channel. And only a few approaches (e.g. [16]-[18]) offer solutions that do not require the exchange of information and therefore they avoid using for control purposes the communication channel of the information and communication technology (ICT) infrastructure that is available in today's MGs [19], [20]. This prevents the degradation that may occurs in distributed control strategies for active power sharing and frequency restoration due to message dropouts, time delays, transmission intervals, quantization, sampling schemes, and traffic scheduling, e.g. [21]-[23].

Focusing on these type of solutions that do not require using the communication channel, this paper presents a dual control approach to be implemented at each VSI that only uses local information for achieving active power sharing and frequency restoration. The starting point is the frequency droop method for active power sharing complemented with a local correction term of the frequency error for frequency restoration. And the correction term is based on an event-driven parametervarying first-order filter of the frequency error that allows for adaptability of the control. A system model that is able to capture the interaction between inverters is also presented, and it is used for assessing stability and for control design.

Adaptability and parameter variation in the droop method (and/or secondary control) have been treated previously (see references in the recent state-of-the-art [24]). For example, the adaptive droop scheme proposed in [25] is designed to ensure active damping of power oscillations at different operating conditions. The control continuously updates an additional proportional control gain in order to maintain the same dynamic characteristics for all operational points. The approach presented in this paper uses a complementary strategy, and adaptivity is applied to force different dynamic characteristics depending on diverse operational conditions. Similarly, eventtriggering mechanisms for power systems have been included in different approaches (e.g., [26]-[30]). However, their joint application bringing together the benefits of both techniques has not been yet explored in this context. By borrowing concepts from [17] and [18], the proposed frequency restoration strategy presents operational improvements that outperform previous works. In particular, it breaks the inherent trade-offs between transient dynamics and accuracy imposed by local proportional controllers and avoids the instability problems that local proportional-integral controllers would otherwise introduce [31]. The operation of the dual control approach sequentially applies two control configurations: a first one that seeks to achieve fast power sharing dynamics and a second one that targets high accuracy for frequency restoration. Therefore, after each execution of the dual control approach, both control 
goals can be successfully achieved without risking instability.

The paper extends the current state-of-the-art of control strategies where the exchange of control data over the ICT infrastructure is not required. The presented dual control approach a) ensures fast active power sharing and high accuracy in the frequency restoration, b) guarantees improved control performance with respect to [18], c) the stability analysis is based on a more accurate modeling effort than the presented in [18] and d) provides similar performance to those approaches using communications. Selected experiments on a laboratory MG show the performance of the dual control approach and its advantages with respect to previous work.

The rest of the paper is structured as follows. Section II introduces the dual control scheme. Section III presents the system model and the stability analysis. Selected experiments are included in Section IV and Section V concludes the paper.

\section{DuAl Control ApProACH}

\section{A. Droop control}

The conventional frequency and voltage droop method locally implemented at each VSI can be expressed as

$$
\begin{aligned}
\omega_{i} & =\omega_{0 i}-m_{i} P_{i} \\
V_{i} & =V_{0 i}-n_{i} Q_{i}
\end{aligned}
$$

where $\omega_{i}$ and $V_{i}$ are the inverter output voltage frequency and amplitude, $\omega_{0 i}$ and $V_{0 i}$ are the reference frequency and amplitude, $P_{i}$ and $Q_{i}$ are the output active and reactive power of the inverter, and $m_{i}$ and $n_{i}$ are the droop proportional control gains. The specification of the gains is a trade-off between transient response and stability.

\section{B. Secondary control via a parameter-varying filter}

The focus for secondary control is on frequency restoration while keeping active power sharing. A secondary voltage control with reactive power sharing is deliberately omitted in this paper because its inclusion would not alter the paper contributions. The strategy presented to remove the frequency deviation is based on adding a corrective term in the frequency droop equation (1) in terms of a time-varying filter of the frequency error. Noting that $P_{i}$ in (1) can be obtained via a first-order low-pass filter as

$$
P_{i}(s)=\frac{\omega_{p}}{s+\omega_{p}} p_{i}(s)
$$

where $s$ is the Laplace operator, $p_{i}(s)$ is the measured instantaneous power at the inverter, and $\omega_{p}$ is the cut-off frequency of active power low-pass filter, the proposed control strategy using (1), (3) and the new time-varying filter can be written in the Laplace domain as

$$
\omega_{i}(s)=\omega_{0 i}(s)-m_{i} \frac{\omega_{p}}{s+\omega_{p}} p_{i}(s)+\frac{\tilde{k} \omega_{s}}{s+\omega_{s}}\left(\omega_{0 i}(s)-\omega_{i}(s)\right)
$$

where $\omega_{s}$ is the additional filter cut-off frequency and $\tilde{k}$ is its time-varying gain. Therefore, the set of parameters to shape the system dynamics includes those of the droop control, $m_{i}$ and $\omega_{p}$, and those of the filter, $\tilde{k}$ and $\omega_{s}$. It is interesting to note that strategy (4) can be transformed into

$$
\omega_{i}(s)=\omega_{0 i}(s)-m_{i}(s) \frac{\omega_{p}}{s+\omega_{p}} p_{i}(s)
$$

with

$$
m_{i}(s)=m_{i} \frac{s+\omega_{s}}{s+(1+\tilde{k}) \omega_{s}} .
$$

Notice that (5) resembles the standard droop control (1) if omitting the active power filter. However, the inverse Laplace transform of (5) leads to the convolution of $m_{i}(t)$ and $p_{i}(t)$ rather than their product, as in the case of the standard droop (1). Hence, the proposed control strategy (4) can not be reduced to a standard droop control, and it offers several advantages that are revealed throughout the paper.

The law-of-variation of the time-varying gain $\tilde{k}$ constitutes the key point for successful operation of the presented control scheme because its performance in terms of frequency restoration and active power sharing highly depends on the value of $\tilde{k}$. Let

$$
e_{\omega}(s)=\omega_{0 i}(s)-\omega_{i}(s)=K_{p}^{\omega} G(s) p_{i}(s)
$$

be the transfer function of the frequency error where $G(s)$ is the canonical transfer function from input $p_{i}(s)$ to output $e_{\omega}(s)$, and $K_{p}^{\omega}$ is the canonical gain. Its calculation leads to

$$
K_{p}^{\omega}=\frac{m_{i}}{1+\tilde{k}}
$$

which clearly depends on $m_{i}$ and $\tilde{k}$, and it is inversely proportional to the filter gain $\tilde{k}$. The value for $m_{i}$ is given by the droop design. Hence, the value of $\tilde{k}$ is the key parameter and its dynamic adjustment will permit achieving small frequency error and fast power transient dynamics.

The desired pattern of variation for $\tilde{k}$ is as follows. Whenever a load or generation change occurs, a change in the active power of each inverter occurs, leading to a transient power unbalance scenario. In this case, a small gain for $\tilde{k}$, namely $k_{\min }$, should start applying because fast correction in the power dynamics is desired to recover perfect sharing. After that, the value of $\tilde{k}$ should increase up to a limit, namely $k_{\max }$, which ensures high accuracy in frequency restoration. And $\tilde{k}$ keeps the $k_{\max }$ value during the time the system is in steady-state until a new load or generation change occurs. An event detection strategy must be designed in order to determine the time at which the $k_{\min }$ value has to be assigned to the filter gain $\tilde{k}$, and a time-driven protocol must be established to specify the law-of-variation of the time-varying gain $\tilde{k}$.

\section{Event-driven condition}

The event condition, which should be locally checked at each VSI and will trigger the time-driven protocol, can be designed in terms of frequency $\omega_{i}$ or active power $p_{i}$, because changes in both magnitudes occur. Henceforth, changes in the active power will be considered. Its design can obey different strategies related to the problem of sampling signals. Among the different event-driven sampling strategies [32], one of the most employed is the level-crossing sampling that mandates to sample a signal when its value has changed by a given 


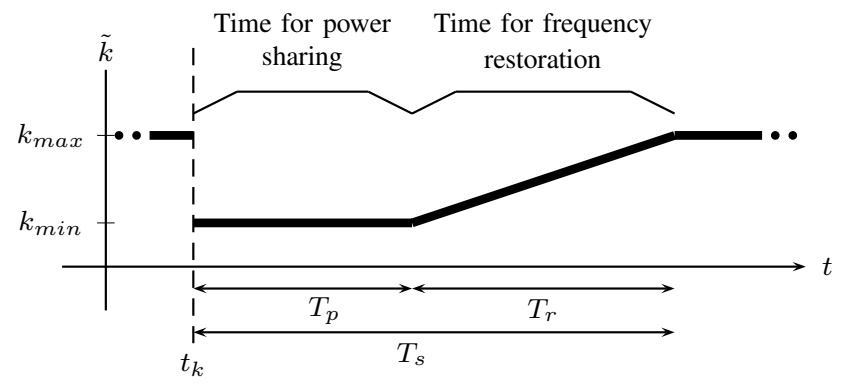

Fig. 1: Time-driven protocol for the filter time-varying gain $\tilde{k}$.

increment with respect to the value of the previous sample. This mechanism has been adapted to a broad spectrum of technology and applications and in the sensor/control networking community it is known as the send-on-delta or deadbands [33].

In the level-crossing sampling, the event-condition is triggered (and an action is taken) if the signal deviates by $\Delta$, defined as a significant change of its value in relation to the most recent sample. The threshold $\Delta$ is a design parameter that determines the resolution of signal observations and the frequency of the taken actions. The smaller threshold $\Delta$, the higher number of actions to be taken. In the presented approach, once the event-condition fires (and the time-driven protocol for varying $\tilde{k}$ starts), it is disabled during a safety time interval to ensure that it will become active again whenever it is expected to have the active power and frequency in steadystate, meaning perfect power sharing and restored frequency.

According to these constraints, the level-crossing mechanism applied in the event-condition is

$$
t_{k}:=\inf \left\{t>t_{k-1}+T_{s}:\left|p_{i}(t)-p_{i}\left(t_{k-1}+T_{s}\right)\right| \geq \Delta p\right\}
$$

where $t \in \mathbb{R}, t_{k} \in \mathbb{R}$ denote the time instants, $\Delta p$ is the threshold around $p_{i}$, and $T_{s}$ is the safety time interval.

\section{Time-driven protocol}

Once the event-condition is triggered, the filter gain $\tilde{k}$ value has to follow a pattern, starting form $k_{\min }$ to $k_{\max }$. This pattern, which is driven by the progression of time, must fulfill three constraints.

First, at the beginning of the pattern, it must be kept $\tilde{k}=k_{\text {min }}$ during enough time, named $T_{p}$, to ensure that power sharing has been successfully achieved. Second, at the end of the pattern, the time varying gain value must be kept to $\tilde{k}=k_{\max }$, which ensures the best accuracy in terms of frequency restoration. Third, the transition of $\tilde{k}$ from $k_{\min }$ to $k_{\max }$ is specified to occur during $T_{r}$ and should be smooth enough to minimize abrupt changes that may damage the electronic equipment. As indicated by later research on frequency restoration [34], the traditional separation of time scales between frequency and active power dynamics seems to be not required, and therefore the specification of $T_{r}$ could be similar to the value given to $T_{p}$.
Summarizing, the time driven protocol for setting the values of $\tilde{k}$ can by specified by

$$
\tilde{k}=\left\{\begin{aligned}
k_{\min } & \text { if } \quad t_{k} \leq t<t_{k}+T_{p} \\
k(t) & \text { if } t_{k}+T_{p} \leq t<t_{k}+T_{p}+T_{r} \\
k_{\max } & \text { if } t_{k}+T_{p}+T_{r} \leq t
\end{aligned}\right.
$$

where $k(t): \mathbb{R} \rightarrow \mathbb{R}$ should be a smooth function that brings $\tilde{k}$ from $k_{\min }$ to $k_{\max }$. The solution adopted in this paper for $k(t)$ is a ramp defined as

$$
k(t)=k_{\min }+\frac{k_{\max }-k_{\min }}{T_{r}}\left(t-t_{k}-T_{p}\right) \quad, \quad t \in \mathbb{R}
$$

The overall time driven protocol (9)-(10) is schematically illustrated in Figure 1, where $t_{k}$ denotes the time at which the event condition fires.

\section{E. Design guidelines}

Regarding the design of the dual control approach (4), apart from the standard $m_{i}$ control gain and the cut-off frequency of the power and frequency filters, $\omega_{p}$ and $\omega_{s}$, the rank of variation of proportional gain $\tilde{k}$ given by $k_{\min }$ and $k_{\max }$ must be analyzed. The $k_{\min }$ parameter determines the maximum allowed frequency error, $e_{\omega, \max }$. Hence, from the frequency error equation (6) and its gain (7) the design of this parameter obeys

$$
k_{\min }=\frac{m_{i} p_{\max }-e_{\omega, \max }}{e_{\omega, \max }}
$$

where $p_{\max }$ is the maximum among all maximum powers that can be delivered by each $i^{\text {th }}$ inverter $p_{i, \max }$, that is, $p_{\max }=\max _{i}\left\{p_{i, \max }\right\}$. Similarly, the $k_{\max }$ parameter determines the desired frequency error $e_{\omega, d}$ at steady-state, and from (6) and (7) its design obeys

$$
k_{\max }=\frac{m_{i} p_{\max }-e_{\omega, d}}{e_{\omega, d}} .
$$

The event condition (8) is characterized by the amount of power variation $\Delta p$ required to fire the time-driven protocol. The threshold $\Delta p$ must be designed to be responsive enough to changes in active power. Increasing the responsiveness demands smaller $\Delta p$. On one hand, a lower bound on $\Delta p$ should consider measurement noise and other small oscillations that may appear in the power that should not fire the event condition. The upper bound for $\Delta p$ depends on the maximum allowed frequency error. From (6), in an equilibrium scenario at time $t_{k}$, the frequency error is the desired one, given by

$$
e_{\omega, d}=\frac{m_{i}}{1+k_{\max }} p_{i}\left(t_{k}\right)
$$

If a change in load occurs, $p_{i}(\cdot)$ starts increasing. Let

$$
e_{\omega, \max }=\frac{m_{i}}{1+k_{\max }} p_{i}(t)
$$

denote the maximum allowed error achieved at time $t>t_{k}$. Subtracting (13) from (14), the maximum error increment is

$$
e_{\omega, \max }-e_{\omega, d}=\frac{m_{i}}{1+k_{\max }}\left(p_{i}(t)-p_{i}\left(t_{k}\right)\right)
$$

which establishes the upper bound for $\Delta p$ as

$$
\Delta p \leq \frac{1+k_{\max }}{m_{i}}\left(e_{\omega, \max }-e_{\omega, d}\right) .
$$


The safety interval of the time driven protocol is defined by $T_{p}$ and $T_{r}$, whose characterization depends on the droop coefficients $m_{i}$ and the system response dynamics. $T_{p}$ is the time that should elapse after the event detection to ensure that power sharing is achieved. Therefore, its specification is related to the desired settling time for the active power, which is given by the dominant pole of the input/output relation between $\omega_{i}$ and $p_{i}$, that depends on $m_{i}$. Hence, in order to achieve faster power sharing dynamics, high values for $m_{i}$ must be specified, and thus short values of $T_{p}$ are required (and vice-versa). However, high values for $m_{i}$ also increase the frequency error as observed from (6)-(7). Luckily, the gain $\tilde{k}$ permits correcting the frequency error to the desired value. Note also that the droop gain $m_{i}$ should not be high in general because as the number of inverters increase, the likelihood of making the system unstable increases [35].

The specification of $T_{r}$ is constrained by $T_{p}$ as $T_{r} \geq T_{p}$. This constraint has the following explanation. A priori, the time interval $T_{r}$ could take any value if ideal conditions for the operation of the dual control approach are assumed. However, when ideal conditions are lost, the value of the time interval $T_{r}$ becomes important. For example, if an anomalous detection of the event occurs (by either a non-detection of the event by one of the inverters or when the event is not detected in a synchronous manner by all inverters), transient errors in power sharing occur (as it will be presented illustrated in Figure 7). In this case, the longer $T_{r}$ is, the better because the shorter and smaller will be the transient errors. In fact, if $T_{r}$ is very short, say instantaneous (meaning that $\tilde{k}$ varies in a step form), the de-synchronicity in the event detection will lead to steps starting at different times. These steps will produce instantaneous phase errors that, due to standard power flow equations, would lead to an instantaneous power sharing error. Although this error will be mitigated by the droop, it will take longer than desired because the droop will be able to start counteracting the error once it has been already created. However, if $T_{r}$ is long, the de-synchronicity will lead to a slow-varying power sharing error that the droop will start counteracting from the beginning, meaning that it will be smaller and it will last shorter. Hence, a compromise specification is to set $T_{r}$ at least equal to the settling time of the active power dynamics, that is, $T_{r} \geq T_{p}$.

Similar to the droop design principle that imposes the same $m_{i}$ value for all inverters in order to achieve power sharing (see eq. (1)), the dual control approach (4) also requires to have the same values for the key design parameters $k_{\text {min }}$, $k_{\max }$ that constraint $\tilde{k}$, for $\Delta p$ that determines the sensitivity of the event condition, and for $T_{p}$ and $T_{r}$ that determine the time driven protocol.

\section{Stability AnALysis}

The stability and transient response analysis of the proposed dual control approach is based on the scheme of the power exchanged between two consecutive nodes. It is applied to the laboratory MG that is characterized by three inverters $G_{1,2,3}$ (enabled with virtual impedances, $Z_{v}$ ), four line impedances $Z_{1,2,3,4}$, and a load impedance $Z_{L}$, as schematically illustrated in Figure 2.

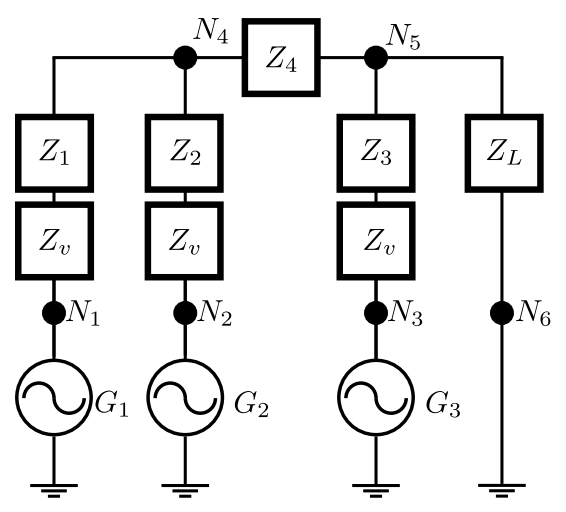

Fig. 2: Circuit diagram of the laboratory $M G$

Using power flow analysis, the power exchange between two nodes is given by

$$
\begin{aligned}
p_{i, j}(t)=V_{i}^{2}(t) g_{i, j} & -V_{i}(t) V_{j}(t) g_{i, j} \cos \left(\varphi_{i}(t)-\varphi_{j}(t)\right) \\
& +V_{i}(t) V_{j}(t) b_{i, j} \sin \left(\varphi_{i}(t)-\varphi_{j}(t)\right)
\end{aligned}
$$

where $\varphi_{i}=\frac{\omega_{i}}{s}$ and the admittance between the two nodes is

$$
g_{i, j}+b_{i, j} j=\frac{1}{R_{i, j}+X_{i, j} j}
$$

From (17), and by using the small-signal analysis, the exchanged power $\hat{p}_{i, j}$ between nodes $i$ and $j$ is given by

$$
\hat{p}_{i, j}=\frac{X_{i, j} V_{i} V_{j}}{s\left(R_{i, j}^{2}+X_{i, j}^{2}\right)}\left(\hat{\omega}_{i}-\hat{\omega}_{j}\right)
$$

where $R_{i, j}$ and $X_{i, j}$ are the equivalent resistance and inductance between nodes $i$ and $j, \omega_{i}$ and $\omega_{j}$ are the nodes frequencies, and $V_{i}$ and $V_{j}$ are the nodes voltage amplitudes in steady-state. It is worth mentioning that the equivalent inductance includes the virtual inductance, if any, plus line inductance.

For mainly inductive distribution lines $\left(R_{i, j} \lll X_{i, j}\right)$, equation (19) simplifies to

$$
\hat{p}_{i, j}=\frac{V_{i} V_{j}}{s X_{i, j}}\left(\hat{\omega}_{i}-\hat{\omega}_{j}\right)
$$

By applying principles (19) and (20) to the laboratory MG and using the compact formalism introduced in [34], the power exchanged between each pair of consecutive nodes $N_{i}$ is

$$
\begin{aligned}
\hat{p}_{1,4}(s) & =\frac{V_{1} V_{4}}{s X_{1,4}}\left(\hat{\omega}_{1}(s)-\hat{\omega}_{4}(s)\right) \\
\hat{p}_{2,4}(s) & =\frac{V_{2} V_{4}}{s X_{2,4}}\left(\hat{\omega}_{2}(s)-\hat{\omega}_{4}(s)\right) \\
\hat{p}_{3,5}(s) & =\frac{V_{3} V_{5}}{s X_{3,5}}\left(\hat{\omega}_{3}(s)-\hat{\omega}_{5}(s)\right) \\
\hat{p}_{4,5}(s) & =\hat{P}_{1,4}(s)+\hat{P}_{2,4}(s)=\frac{V_{4} V_{5}}{s X_{4,5}}\left(\hat{\omega}_{4}(s)-\hat{\omega}_{5}(s)\right) \\
\hat{p}_{5,6}(s) & =\hat{P}_{1,4}(s)+\hat{P}_{2,4}(s)+\hat{P}_{3,5}(s) \\
& =\frac{X_{5,6} V_{5} V_{6}}{s\left(R_{5,6}^{2}+X_{5,6}^{2}\right)}\left(\hat{\omega}_{i}-\hat{\omega}_{j}\right)
\end{aligned}
$$

where $X_{1,2}=X_{v}+X_{1}, X_{2,4}=X_{v}+X_{2}, X_{3,5}=X_{v}+X_{3}$, $X_{4,5}=X_{4}, R_{5,6}=R_{L}$ and $X_{5,6}=X_{L}$ as deduced from 
Figure 2 . Note that the active power delivered by $G_{1}, G_{2}$, and $G_{3}$ is $\hat{P}_{1,4}, \hat{P}_{2,4}$ and $\hat{P}_{3,5}$ respectively, that from now on they will be denoted as $\hat{P}_{1}, \hat{P}_{2}$ and $\hat{P}_{3}$. By solving the system of equations (21) and assuming $V_{i}=V_{j}=V$, the plant model is obtained

$$
\hat{P}(s)=G(s) \hat{\Omega}(s)
$$

where $G(s)$ is a matrix of transfer functions obtained from (21).

For each node, the dual control strategy (4) in terms of the small signal variables is given by

$$
\begin{aligned}
\hat{\omega}_{i}(s) & =\hat{\omega}_{0 i}(s)-m_{i} \frac{\omega_{p}}{s+\omega_{p}} \hat{P}_{i}(s)+\frac{\tilde{k} \omega_{s}}{s+\omega_{s}}\left(\hat{\omega}_{0 i}(s)-\hat{\omega}_{i}(s)\right) \\
& =\hat{\omega}_{0 i}(s)-\frac{m_{i}}{1+\frac{k \omega_{s}}{s+\omega_{s}}} \frac{\omega_{p}}{s+\omega_{p}} \hat{P}_{i}(s) \\
& =\hat{\omega}_{0 i}(s)-h(s) \hat{P}_{i}(s)
\end{aligned}
$$

Note that in (23) the perturbation $\hat{\omega}_{0 i}$ on the nominal frequency $\omega_{0 i}$ is specific for each inverter.

For the laboratory MG (Figure 2), the feedback control (23) in compact form is given by

$$
\hat{\Omega}(s)=\hat{\Omega}_{0}(s)-H(s) \hat{P}(s)
$$

By applying the feedback term (24) into the plant (22) the obtained closed-loop compact form is given by

$$
\hat{P}(s)=G(s)\left[\hat{\Omega}_{0}(s)-H(s) \hat{P}(s)\right]
$$

that, after rearranging terms, can be written as

$$
\hat{P}(s)=[I+G(s) H(s)]^{-1} G(s) \hat{\Omega}_{0}(s)
$$

The analysis of the MG stability and transient response can be performed by looking at the location of the closed-loop poles of (26) as a function of $\tilde{k}$ in $H(s)$. The parameters shown in Table I have been used in the root-locus analysis. Their values have been chosen to match those also used in the laboratory experiments. Figure 3 shows the root locus for four out of the six poles of the system, namely $\lambda_{1}-\lambda_{4}$. The remaining two poles do not appear in the figure because they are located far away to the left of the imaginary axis. The figure shows the location of three sets of four poles corresponding to three different values of the gain $\tilde{k}$. The first set marked with triangles corresponds to the cases when only droop applies, $\tilde{k}=0$. The other two sets, marked with squares and asterisks, correspond to the case when the frequency restoration filter applies with a gain of $\tilde{k}=1.5$ or $\tilde{k}=20$, respectively.

Looking at the case when only droop applies $(\tilde{k}=0$, triangle markers), the dominant poles have complex conjugate parts different than zero. Therefore, the power sharing dynamics will exhibit a transient oscillation. When the frequency restoration filter applies (squares and asterisk markers), the location of the dominant poles is determined by the value of the filter gain $\tilde{k}$. As the value increases, the dominant poles reduce their complex part (see e.g. $\tilde{k}=1.5$, square markers), up to the point that they become just real (around $\tilde{k}=2$ ), and therefore, the oscillation will disappear. Note also that from
TABLE I: System and control parameters.

\begin{tabular}{c|c}
\hline \hline Parameter & Value \\
\hline$V_{i}$ & $110 \sqrt{3} \mathrm{~V}$ \\
$\omega_{0}$ & $2 \pi 60 \mathrm{rad} / \mathrm{s}$ \\
$Z_{v}$ & $j 3.76 \Omega$ \\
$Z_{1}$ & $0.5+j 1.13 \Omega$ \\
$Z_{2}$ & $0.5+j 0.37 \Omega$ \\
$Z_{3}$ & $1.1+j 0.22 \Omega$ \\
$Z_{4}$ & $j 0.30 \Omega$ \\
$Z_{L}$ & $22 \Omega$ \\
$\omega_{p}$ & $2 \pi \mathrm{rad} / \mathrm{s}$ \\
$\omega_{s}$ & $10 \cdot 2 \pi \mathrm{rad} / \mathrm{s}$ \\
$m_{i}$ & $1 \mathrm{mrad} /(\mathrm{Ws})$. \\
$n_{i}$ & $0.5 \mathrm{mV} /(\mathrm{VAr})$. \\
$\tilde{k}$ & {$[0,20]$} \\
\hline \hline
\end{tabular}

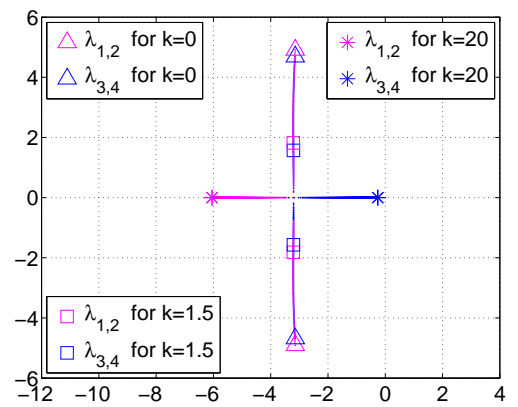

Fig. 3: Root locus varying $\tilde{k}$.

this point, the pair of poles separate, and one goes toward the imaginary axis, thus imposing a slower dynamics (see e.g. $\tilde{k}=20$, asterisk markers). This corroborates the design guidelines introduced in Section II-B where it was assumed that fastest transient responses are achieved only for small values of $\tilde{k}$.

It is important to note that the dynamic model used in the stability analysis is a simplified form of the full model of an inverter provided in the literature, e.g. [36]. The simplification is due to the fact that the inner current and voltage loops have not been considered because they are sufficiently fast compared to the proposed control that they would not alter the imposed dominant dynamics. In addition, the analyzed model considers that the voltages phases are similar enough that their difference can be approximated by zero, as it is commonly assumed in modeling approaches involving mainly inductive MGs [37].

\section{EXPERIMENTAL RESULTS}

This section presents selected experimental results from a small-scale laboratory microgrid that illustrate the operation and properties of the dual-control approach.

Figure 4 shows the diagram of the three nodes low power MG that has been built in the laboratory whose main parameters are summarized in Table I. Each node consists of a $2 \mathrm{kVA}$ three-phase full-bridge power inverter MTLCBI0060F12IXHF from GUASCH, working at $10 \mathrm{kHz}$, and connected in parallel to a resistive load $L_{1,2,3}=500 \mathrm{~W}$ (0 VAr). A resistive bus load named $L_{\text {bus }}$ (characterized by 


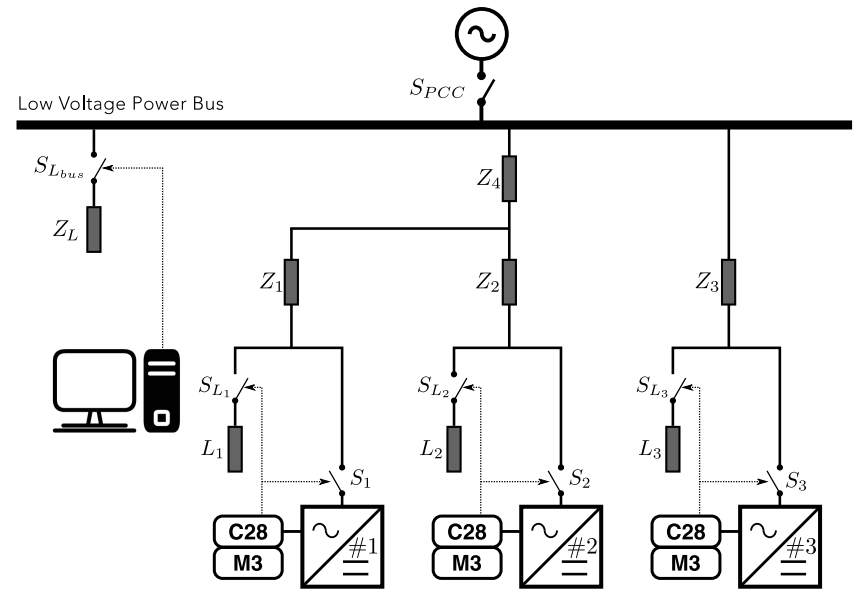

Fig. 4: Scheme for the laboratory microgrid.

$Z_{L}$ in Table I) is also available. An AMREL SPS-800-12 dc power source has been used to emulate distributed generation sources that supply the inverters. Each inverter is connected to the MG via an $L C$ filter $(5 \mathrm{mH}, 1.5 \mu \mathrm{F})$ and a wye-delta transformer. The transformer parasitic resistance/inductance (resistance/inductance $=0.5 \Omega / 3 \mathrm{mH}$ in VSI number $1,0.5$ $\Omega / 1 \mathrm{mH}$ in VSI number 2 , and $1.1 \Omega / 0.6 \mathrm{mH}$ in VSI number 3) and physical three-phase inductances have been used to emulate the distribution lines. The line impedances vary from more inductive to more resistive. However, the inclusion of the virtual impedance at each inverter provokes that the "seen" impedances from the inverters are mainly inductive. In general terms, the virtual impedance control can be designed to choose the suitable power-sharing strategy given the possibly different $X / R$ ratios of the MG lines characteristics [38]. Each inverter controller is based on a dualcore Texas Instruments Concerto board consisting in a C28 floating point digital signal processor (DSP) that implements the control algorithm and a M3 communications processor that is only used for monitoring purposes.

The majority of the experiment runs follow the same simple pattern over 60 s although some figures include executions up to 120 s in order to provide a reacher set of patterns. First of all, the bus load $L_{b u s}$ is always active while the other loads $L_{1,2,3}$ are always inactive. At time $t=0 \mathrm{~s}$, generator number 1 becomes active, at time $t=20$ s generator number 2 becomes active, and at time $t=40$ s generator number 3 becomes active. Whenever the experiment starts, the control is active during the whole execution time. In the activation of each generator, a phase-locked loop (PLL) starts operating right before connecting into the grid in order to eliminate the phase error and guarantee a smooth connection. Whenever the experiment follows another pattern, it will be described.

\section{A. Proposed droop control with parameter-varying filter}

Figure 5 illustrates the main paper contribution achieved by the dual control approach where both fast and accurate active power and frequency dynamics are achieved. In particular, it shows the application of the dual control approach (4) with a time varying gain $\tilde{k}$ ranging from $k_{\min }=2.5$ to $k_{\max }=20$.
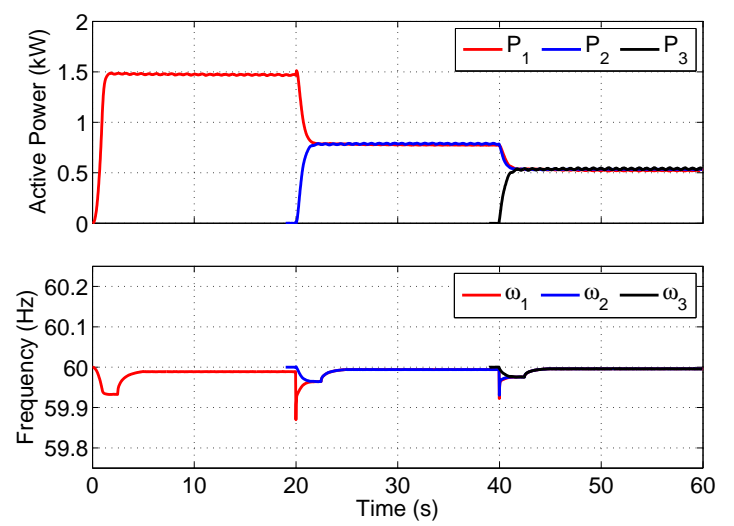

Fig. 5: Active power and frequency for the three VSI with time-varying filter with gain $\tilde{k}$ varying from 2.5 to 20 .

The event condition and the time driven protocol have been applied. The threshold is defined in such a way that the event condition fires when the active power varies more than $10 \%$ the nominal VSI power. And the time intervals $T_{p}$ and $T_{r}$ of the time driven protocol are set to $2.5 \mathrm{~s}$.

Looking at the active power sub-figure (top of Figure 5), it can be observed that the dynamics are fast, thus permitting to shape the dynamics to fulfill stringent requirements. Looking at the frequency restoration sub-figure (bottom of Figure 5), it can be observed that the presented approach permits also to achieve a high level of accuracy. And both control goals can be fulfilled without requiring communications between VSI. Hence, the proposed approach solves the trade-off between transient speed in the active power sharing and steady-state accuracy in the frequency restoration. This is achieved at the expenses of the punctual deviations that appear during transients in the inverters output frequency (bottom of Figure 5, times $t=20 \mathrm{~s}$ and $t=40 \mathrm{~s})$. However, such deviations can be perfectly accepted by inverters as long as the frequency remains within desired limits.

\section{B. Performance evaluation}

The first set of figures illustrate the design tradeoff that is imposed by the existing state-of-the-art droop control with static filter. In particular, Figure 6 shows the active power and frequency dynamics for the three VSI with droop control and frequency restoration filter with static gain for two specific values of $\tilde{k}(\tilde{k}=2.5$ in sub-figure $6 \mathrm{a}$ and $\tilde{k}=20$ in subfigure $6 \mathrm{~b}$ ) corresponding to the limits of the varying range illustrated previously in Figure 5 . In both cases the droop gain is as before, $m_{i}=1 \mathrm{mrad} /(\mathrm{Ws})$. In sub-figure 6a the power sharing exhibits fast dynamics (the transient is less than $2.5 \mathrm{~s}$ ) but the frequency exhibits an noticeable error, ranging from $0.03 \mathrm{~Hz}$ to $0.1 \mathrm{~Hz}$. Complementary, in the sub-figure $6 \mathrm{~b}$, the frequency restoration is more accurate, with a negligible error less than $5 \mathrm{mHz}$, at the expenses of imposing slower dynamics (the transient is around 20s), which may not be admissible. Therefore, the state-of-the-art solution [17] without using communications does not offer a solution permitting 



(a) With static filter gain $\tilde{k}=2.5$
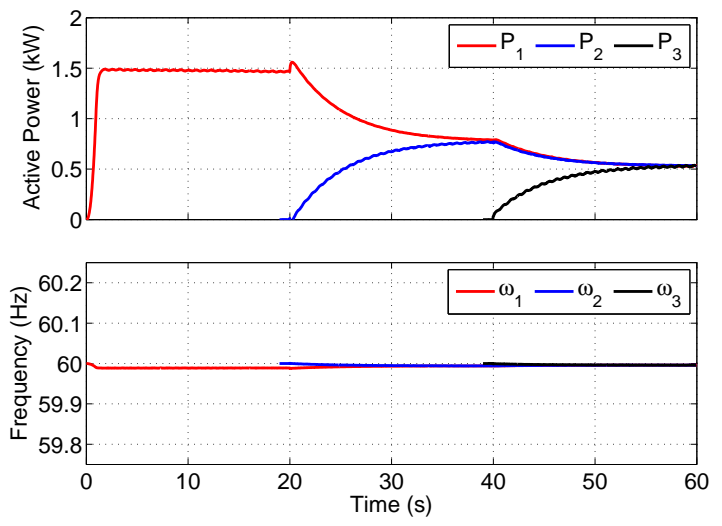

(b) With static filter gain $\tilde{k}=20$

Fig. 6: Active power and frequency for the three VSI with static filter gain $\tilde{k}$
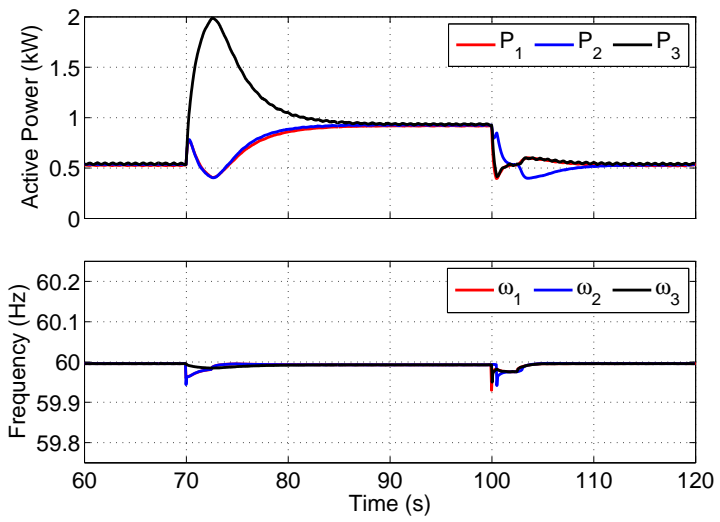

Fig. 7: Anomalous triggering of the event condition: inverter $G_{3}$ does not detect a step load change at time $t=70 \mathrm{~s}$ and inverter $G_{2}$ at time $t=100$ s detects a step load change $0.5 \mathrm{~s}$ later than $G_{1}$ and $G_{3}$.

the fulfillment of both control goals with a high degree of satisfaction as obtained by the dual control approach.

The second evaluation analyses the performance in the case of desynchronized triggering of the event condition that serves as a comparison with respect to [18], which is also based on an event-driven operation of a parameter-varying filter. On the one hand, the approach in [18] offers degraded performance for desynchronized triggering of the event condition in the sense that power sharing is lost. Figure 7 shows the performance of the dual control approach for different anomalous triggering of the event condition. Two scenarios are evaluated. The first one corresponds to the case where the third inverter $G_{3}$ does not detect a load change at time $t=70 \mathrm{~s}$. As it can observed in the active power top sub-figure, slow and oscillatory dynamics dominate instead of the desired fast ones. The second scenario corresponds to the desynchronized triggering of the event condition where the second inverter $G_{2}$ detects 0.5 s later than $G_{1}$ and $G_{3}$ a load change at time $t=100$ s. As it can be observed in the top subfigure, the active powers follow also a slow and oscillatory dynamics. Hence, failures in the event detection cause unexpected dynamics for the active power, but the system is still driven to the desired steady-state values achieving perfect power sharing and frequency restoration.

The third evaluation focuses on the case where the MG is subject to multiple load changes, which also serves for performance comparison with respect to [18]. The dual control approach is based on disabling the event detection during the safety interval while the time driven protocol is active to ensure meeting the control goals within the predefined time bounds. Hence, it is of interest to analyze the performance when changes in the MG occur often enough that some of them take place while the event detection is disabled. Figure 8 shows the case of load changes occurring during time intervals $T_{p}$ or $T_{r}$, that constitute the safety interval. In particular subfigure 8 a covers the scenario where load $L_{1}$ is active from the beginning and it is disconnected at $t=21 \mathrm{~s}$, that is, $1 \mathrm{~s}$ after the time interval $T_{p}$ has started due to the connection of $G_{2}$ at time $t=20 \mathrm{~s}$. During $T_{p}$, the dual control approach mainly acts on achieving power sharing with fast dynamics. Hence, during this time interval, a change of load will be correctly and immediately addressed by the control, as it can be observed in sub-figure 8a. Sub-figure 8b shows a similar scenario when load $L_{1}$ is connected again at time $t=43.5 \mathrm{~s}$, that is, $1 \mathrm{~s}$ after the time interval $T_{r}$ has started due to the connection of $G_{3}$ at time $t=40 \mathrm{~s}$. During $T_{r}$, the dual control approach is devoted to fast frequency restoration. Hence, the power sharing dynamics will reach the desired set-point but exhibiting a transient that may not meet the desired specifications, as it can be identified in sub-figure $8 \mathrm{~b}$. In both cases (small) changes in the active power dynamics can be observed while these changes can hardly be appreciated in the frequency, which indicates that the dual control approach is able to deal with these situations in a satisfactory manner. This is an advantage with respect to the approach by [18] where frequency and power sharing errors last longer than desired because the event detection in [18] is permanently active.

The last evaluation serves to qualitatively asses the performance losses that occur when avoiding using communications. To do so, two cases where the inverters apply a secondary control policy that requires the exchange of control data over a communication network are reported. The evaluated approaches are a standard centralized approach [2] and a 

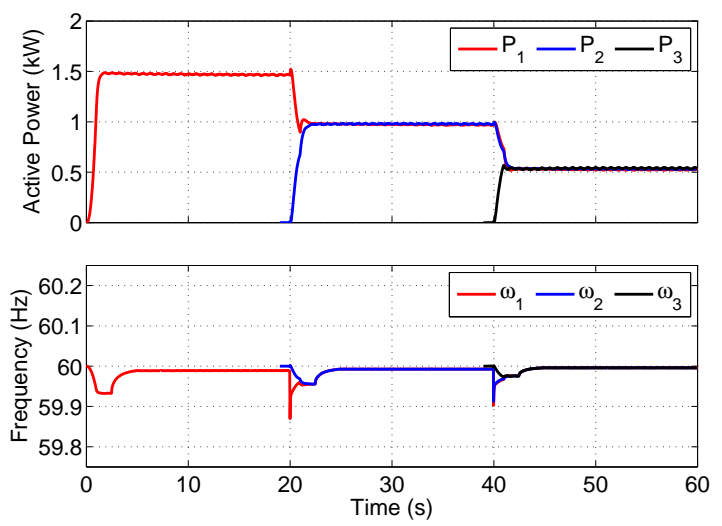

(a) Changes during $T_{p}$.


(b) Changes during $T_{r}$

Fig. 8: Connection and disconnection of a load during $T_{p}$ or $T_{r}$ : load $L_{1}$ is disconnected and connected 1 s after time intervals $T_{p}$ or $T_{r}$ have started due to the connection of G2 and $\mathrm{G} 3$, respectively.

distributed approach inspired on a consensus-based control strategy [8], both to be compared with the ones obtained by the dual control approach in Figure 5. Sub-figures $9 a$ and $9 b$ show the corresponding active power and frequency dynamics for the centralized and consensus approaches, respectively. It can be appreciated that both achieve a fast transient response for the active power sharing and zero-error steady-state for frequency restoration (in spite of exhibiting also punctual deviations in the frequency like those occurring in the dual control approach). In terms of control performance, the centralized and the consensus-based secondary control outperforms the dual control approach that inevitably introduces acceptable control degradation with the benefit of not requiring communications.

\section{CONCLUSiON}

This paper has presented a novel dual control approach for active power sharing and accurate frequency regulation for parallel VSI working in islanded mode. It is based on a design principle that, upon detection of a load or generation change, schedules control actions following a time-driven protocol that permits smoothly change the goal of the control action
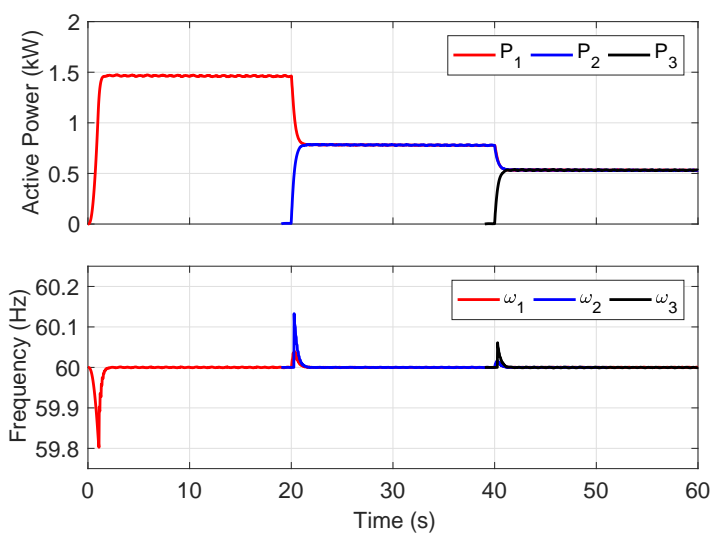

(a) Centralized-based secondary control.
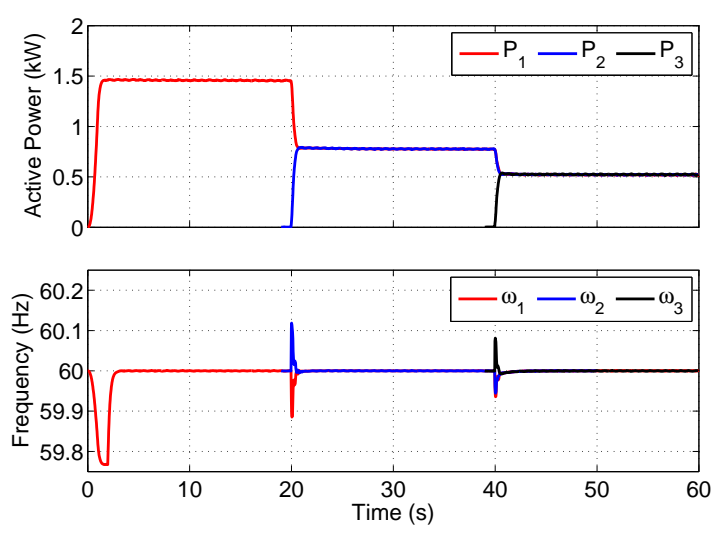

(b) Consensus-based secondary control

Fig. 9: Active power and frequency for the three VSI with communication-based secondary control.

to achieve both negligible frequency error and fast active power responses. A key feature of the presented approach relies on the fact that control goals are achieved without intercommunications between VSI. Experimental results have corroborated the benefits of the presented dual approach and its advantages with respect to previous work or standard communication-based solutions.

\section{REFERENCES}

[1] M.C. Chandorkar, D.M. Divan and R. Adapa, R., "Control of parallel connected inverters in standalone AC supply systems," EEE Trans. Industry Applications, vol.29, no.1, pp.136-143, Jan/Feb 1993.

[2] J.M. Guerrero, J.C. Vasquez, J. Matas, L. Garcia de Vicuña and M. Castilla, "Hierarchical control of droop-controlled AC and DC microgrids - A general approach toward standardization," IEEE Trans. Industrial Electronics, vol. 58, no. 1, pp. 158-172, Jan. 2011.

[3] Q. Shafiee, J.M. Guerrero and J.C. Vasquez, "Distributed secondary control for islanded microgrids - A novel approach," IEEE Trans. Power Electronics, vol.29, no.2, pp.1018-1031, Feb. 2014.

[4] A. Bidram, A. Davoudi, F.L. Lewis, and S. Sam Ge, "Distributed adaptive voltage control of inverter-based microgrids," IEEE Trans. Energy Conversion, vol. 29, no. 4, pp. 862-872, Dec. 2014.

[5] W. Liu, W. Gu, W. Sheng, X. Meng, Z. Wu, W. Chen, "Decentralized multi-agent system-based cooperative frequency control for autonomous microgrids with communication constraints," IEEE Trans. Sustainable Energy, vol.5, no.2, pp.446-456, 2014 
[6] M.A. Mahmud, M.J. Hossain, H.R. Pota and A.M.T. Oo, "Robust nonlinear distributed controller design for active and reactive power sharing in islanded microgrids," IEEE Trans. Energy Conversion, vol. 29, no. 4, pp. 893-903, Dec. 2014

[7] C. Gavriluta, I. Candela, A. Luna, A. Gomez-Exposito and P. Rodriguez, "Hierarchical control of HV-MTDC systems with droop-based primary and OPF-based secondary," IEEE Trans. Smart Grid, vol.6, no.3, pp.15021510, May 2015

[8] F. Guo, C. Wen, J. Mao, and Y.-D. Song, "Distributed secondary voltage and frequency restoration control of droop-controlled inverter-based microgrids," IEEE Trans. Industrial Electronics, vol. 62, no. 7, pp. 43554364, July 2015

[9] L.-Y. Lu and C.-. Chu "Consensus-based secondary frequency and voltage droop control of virtual synchronous generators for isolated AC microgrids", IEEE Journal on Emerging and Selected Topics in Circuits and Systems, vol. 5, no. 3, pp. 443 - 455, Sept. 2015

[10] F. Guo, C. Wen, J. Mao, J. Chen, Y.-D. Song, "'Distributed cooperative secondary control for voltage unbalance compensation in an islanded microgrid," IEEE Trans. Industrial Informatics, vol.11, no.5, pp.10781088, Oct. 2015

[11] J.W. Simpson-Porco, Q. Shafiee, F. Dorfler, J.C. Vasquez, J.M. Guerrero and F. Bullo, "Secondary frequency and voltage control of islanded microgrids via distributed averaging," IEEE Trans. Industrial Electronics, vol.62, no.11, pp.7025-7038, Nov. 2015

[12] J. Lai, H. Zhou, X. Lu, X. Yu and W. Hu, "Droop-based distributed cooperative control for microgrids with time-varying delays," IEEE Trans. Smart Grid, vol. 7, no. 4, pp. 1775-1789, July 2016

[13] M.S. Golsorkhi, D.D.C. Lu and J.M. Guerrero, "A GPS-based decentralized control method for Islanded microgrids," IEEE Trans. Power Electronics, vol. 32, no. 2, pp. 1615-1625, Feb. 2017

[14] N.M. Dehkordi, N. Sadati and M. Hamzeh, "Fully distributed cooperative secondary frequency and voltage control of islanded microgrids," IEEE Trans. Energy Conversion, vol. 32, no. 2, pp. 675-685, June 2017.

[15] F.D. Mohammadi, H.K Vanashi and A. Feliachi, "State-space modeling, analysis, and distributed secondary frequency control of isolated microgrids", IEEE Trans. Energy Conversion, vol. 33, no. pp. 155 - 165, 2018.

[16] F. Katiraei and M.R. Iravani,, "Power management strategies for a microgrid with multiple distributed generation units," IEEE Trans. Power Systems, vol.21, no.4, pp.1821-1831, Nov. 2006.

[17] M. Hua, H. Hu, Y. Xing and J.M. Guerrero, "Multilayer control for inverters in parallel operation without intercommunications," IEEE Trans. Power Electronics, vol.27, no.8, pp.3651-3663, 2012.

[18] J.M. Rey, P. Martí, M. Velasco, J. Miret and M. Castilla, "Secondary switched control with no communications for islanded microgrids," IEEE Trans. Industrial Electronics, vol. 64, no. 11, pp. 8534-8545, Nov. 2017.

[19] Q. Yang, J.A. Barria and T.C. Green, "Communication infrastructures for distributed control of power distribution networks," IEEE Trans. Industrial Informatics, vol. 7, no. 2, pp. 316-327, May 2011.

[20] V.C. Gungor et al., "A survey on smart grid potential applications and communication requirements," IEEE Trans. Industrial Informatics, vol. 9, no. 1, pp. 28-42, Feb. 2013.

[21] S. Liu, X. Wang and P.X. Liu, "Impact of communication delays on secondary frequency control in an islanded microgrid", IEEE Trans. Industrial Electronics, pp. 2021 - 2031, vol: 62, num. 4, April 2015.

[22] C. Ahumada, R. Cárdenas, D. Sáez and J.M. Guerrero, ”Secondary control strategies for frequency restoration in islanded microgrids with consideration of communication delays", IEEE Trans. Smart Grid, vol. 7, no. 3, pp. 1430-1441, May 2016

[23] P. Martí, M. Velasco, E.X. Martín, L. García de Vicuña, J. Miret and M. Castilla, "Performance evaluation of secondary control policies with respect to digital communications properties in inverter-based islanded microgrids", in IEEE Trans. Smart Grid, vol. 9, no. 3, pp. 2192-2202, May 2018.

[24] Y. Han, H. Li, P. Shen, E.A.A. Coelho and J.M. Guerrero, "Review of active and reactive power sharing strategies in hierarchical controlled microgrids," IEEE Trans. Power Electronics, vol. 32, no. 3, pp. 24272451, March 2017.

[25] Y.A.I. Mohamed and E.F. El-Saadany, "Adaptive decentralized droop controller to preserve power sharing stability of paralleled inverters in distributed generation microgrids," IEEE Trans. Power Electronics, vol. 23, no. 6, pp. 2806-2816, Nov. 2008.

[26] S. Wen, X. Yu, Z. Zeng and J. Wang, "Event-triggering load frequency control for multiarea power systems with communication delays," IEEE Trans. Industrial Electronics, vol. 63, no. 2, pp. 1308-1317, Feb. 2016.

[27] C. Li, X. Yu, W. Yu, T. Huang and Z. W. Liu, "Distributed eventtriggered scheme for economic dispatch in smart grids," IEEE Trans. Industrial Informatics, vol. 12, no. 5, pp. 1775-1785, Oct. 2016.
[28] L. Dong, Y. Tang, H. He and C. Sun, ”An event-triggered approach for load frequency control with supplementary ADP," IEEE Trans. Power Systems, vol. 32, no. 1, pp. 581-589, Jan. 2017.

[29] C. Dou, D. Yue and J. M. Guerrero, "Multiagent system-based eventtriggered hybrid controls for high-security hybrid energy generation systems," IEEE Trans. Industrial Informatics, vol. 13, no. 2, pp. 584 594, April 2017.

[30] J. Zhou, H. Zhang, Q. Sun, D. Ma and B. Huang, "Event-based distributed active power sharing control for interconnected AC and DC microgrids," IEEE Trans. Smart Grid, vol. 9, no. 6, pp. 6815-6828, Nov. 2018.

[31] M. Andreasson, D. V. Dimarogonas, H. Sandberg and K.H. Johansson, "Distributed control of networked dynamical systems: Static feedback, integral action and consensus", IEEE Trans. Automatic Control, vol. 59 , no. 7 , pp. $1750-1764,2014$.

[32] W.P.M.H. Heemels, K.H. Johansson and P.Tabuada, "Event-triggered and self-triggered control", Encyclopedia of Systems and Control (J. Baillieul and Samad, T. - Eds.), Springer, p. 384-391, 2015.

[33] M. Miskowicz M, "Send-on-delta concept: an event-based data reporting strategy," Sensors, vol. 6, no. 1, pp. 49-63, 2006

[34] V. Nasirian, Q. Shafiee, J.M. Guerrero, F.L. Lewis and A. Davoudi, "Droop-free distributed control for AC microgrids," IEEE Trans. Power Electronics, vol. 31, no. 2, pp. 1600-1617, Feb. 2016.

[35] J.M. Guerrero, L.Garcí de Vicuña, J. Matas, M. Castilla and J. Miret, "A wireless controller to Enhance Dynamic Performance of Parallel Inverters in Distributed Generation Systems," IEEE Trans. Power Electronics, vol. 19 , no. 5, pp. 1205-1213, Sept. 2004.

[36] N. Pogaku, M. Prodanovic, and T. C. Green, "Modeling, analysis and testing of autonomous operation of an inverter-based microgrid," IEEE Transactions on Power Electronics, vol. 22, no. 2, pp. 613?625, March 2007.

[37] J. Schiffer, D. Zonetti, R. Ortega, A.M. Stanković, T. Sezi, and J. Raisch, "A survey on modeling of microgrids -From fundamental physics to phasors and voltage sources," Automatica, vol. 74, pp. 135-150, Dec. 2016.

[38] J.M. Guerrero, L. García de Vicuna, J. Matas, M. Castilla and J. Miret, "Output impedance design of parallel-connected UPS inverters with wireless load-sharing control," IEEE Trans. Industrial Electronics, vol. 52, no. 4, pp. 1126-1135, Aug. 2005.



Juan Rey was born in Bucaramanga, Colombia in 1989. He received the B.S. in electrical engineering from Universidad Industrial de Santander, Bucaramanga, Colombia, in 2012. He is currently working toward the Ph.D. degree in electronic engineering with the Department of Electronic Engineering, Technical University of Catalonia, Spain.

Since 2013, he has been with the Electrical, Electronic and Telecommunications Engineering School (E3T), Universidad Industrial de Santander, Bucaramanga Colombia, where he is currently an Assistan Professor. His research interest include power electronics and control for distributed generation and microgrids.



Carlos Xavier Rosero received the B.Sc. degree in automotive electronics engineering from the Army Polytechnic School, Sangolqui, Ecuador in 2008, and the M.Sc. degree in automatic control and robotics from the Technical University of Catalonia (UPC), Barcelona-Spain in 2015. He is currently working toward the Ph.D. degree in automatic control, robotics and vision from UPC. His research interests are centered on real-time control systems and microgrids with emphasis on control strategies. 


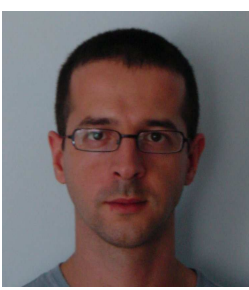

Manel Velasco graduated in maritime engineering in 1999 and received the $\mathrm{PhD}$ degree in automatic control in 2006, both from the Technical University of Catalonia, Barcelona, Spain. Since 2002, he has been an assistant professor in the Department of Automatic Control at the Technical University of Catalonia. His research interests include artificial intelligence, real-time control systems, and collaborative control systems, especially on redundant controllers and multiple controllers with self-interacting systems.

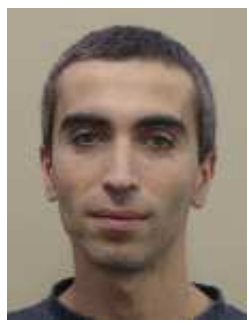

Pau Martí received the degree in computer science and the $\mathrm{PhD}$ degree in automatic control from the Technical University of Catalonia, Barcelona, Spain, in 1996 and 2002, respectively. During 2003 he held a research fellow appointment in the Computer Science Department at the University of California at Santa Cruz, US. From 1996 to 2013, he has been an assistant professor in the Department of Automatic Control at the Technical University of Catalonia. Since 2013 he has been an Associate Professor in the Department of Automatic Control at the Technical University of Catalonia. His research interests include embedded and networked control systems, nonlinear control and microgrids.

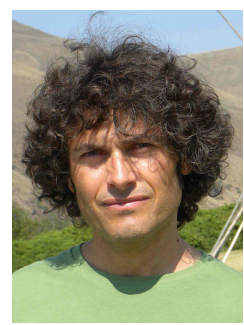

Jaume Miret (M'98) received the B.S. degree in telecommunications, M.S. degree in electronics, and $\mathrm{Ph} . \mathrm{D}$. degree in electronics from the Universitat Politecnica de Catalunya, Barcelona, Spain, in 1992 , 1999, and 2005, respectively. From 1993 to 2011, he was an Assistant Professor in the Department of Electronic Engineering, Universitat Politecnica de Catalunya, Spain. Since 2011 he has been an Associate Professor in the Universitat Politecnica de Catalunya, where he teaches courses on digital design and circuit theory. His research interests include dc-to-ac converters, active power filters, and digital control.



Miguel Castilla received the B.S., M.S. and Ph.D. degrees in telecommunication engineering from the Technical University of Catalonia, Barcelona, Spain, in 1988, 1995, and 1998, respectively. Since 2002, he has been an Associate Professor in the Department of Electronic Engineering, Technical University of Catalonia, where he teaches courses on analog circuits and power electronics. His research interests are in the areas of power electronics, nonlinear control, and renewable energy systems. 